\title{
Mechanisms of Tactile Information Transmission through Whisker Vibrations
}

\author{
Eran Lottem and Rony Azouz \\ Department of Physiology and Neurobiology, Faculty of Health Sciences, Zlotowski Center for Neuroscience, Ben-Gurion University, Beer-Sheva, \\ Israel 84105
}

In their natural environment, rodents use their whiskers to locate and distinguish between objects of different textures and shapes. They do so by moving their whiskers actively as well as passively, through body and head movements. To determine the mechanisms by which surface coarseness is translated into neuronal discharges through passive whisker movements, we monitored head movements of awake behaving rats while approaching objects. We then replayed these movements in anesthetized rats, monitored the whiskers' movements across various surfaces, and concurrently recorded the activity of first-order sensory neurons. We found that whiskers, being the first stage of sensory information translation, shape transduction by amplifying small-amplitude high-frequency signals. Thus, surface coarseness is transmitted through high-velocity micromotions. Consistent with this, we find that during surface contact, discrete highvelocity movements, or stick-slip events, evoke first-order neuronal discharge. Transient ringing in whiskers, which primarily represents resonance vibrations, follows these events, but seldom causes neurons to discharge. These sensory transformations are influenced by the whiskers' biomechanical properties. To determine the resemblance of these tactile transformations during passive whisker movements and active whisking, we induced artificial whisking across various surface textures. We found that the processes by which tactile information becomes available to the animal are similar for these different modes of behavior. Together, these findings indicate that the temporal bandpass properties for spike generation in first-order neurons are matched by the biomechanical characteristics of whiskers, which translate surface coarseness into high-frequency whisker micromotions. These properties enable rodents to acquire tactile information through passive and active movements of their whiskers.

\section{Introduction}

In the rat whisker somatosensory system, tactile information is acquired by an array of whiskers on the facial mystacial pad. Rats actively sweep their whiskers across surfaces in a rhythmic forward and backward motion, called whisking, to locate and distinguish objects in the animals' immediate sensory environment (Sachdev et al., 2001; Bermejo et al., 2002; Berg and Kleinfeld, 2003; Knutsen et al., 2005). Additionally, active whisking movements are always associated with head and body movements as well (Carvell and Simons, 1990, 1995; Brecht et al., 1997; Ritt et al., 2008). Moreover, rodents often forego whisking, relying solely on passive movement of their whiskers instigated by body and head movements. Specifically, they use their vibrissae but do not whisk as they maintain contact with walls and surfaces (Milani et al., 1989; Carvell and Simons, 1990, 1995; Brecht et al., 1997; Towal and Hartmann, 2006; Ritt et al., 2008) while running and when performing an aperture-size (Krupa et al., 2001) task.

Several studies demonstrated that by using their whiskers, rats could reliably detect small differences in surface coarseness

\footnotetext{
Received Feb. 11, 2009; revised June 7, 2009; accepted July 3, 2009.

This work was supported by a grant from the Israel Science Foundation to R.A. We thank Emanuel Lottem for his valuable comments on an earlier version of this manuscript.

Correspondence should be addressed to Dr. Rony Azouz, Department of Physiology and Neurobiology, Faculty of Health Sciences, Zlotowski Center for Neuroscience, Ben-Gurion University, Beer-Sheva, Israel 84105. E-mail: razouz@bgu.ac.il.

D01:10.1523/JNEUROSCI.0705-09.2009

Copyright $\odot 2009$ Society for Neuroscience $\quad$ 0270-6474/09/2911686-12\$15.00/0
}

(Guic-Robles et al., 1989, 1992; Carvell and Simons, 1990, 1995; Lottem and Azouz, 2008). Two mutually exclusive theories have been suggested to underlie this capability. The resonance hypothesis posits that texture identity is represented spatially across the whisker pad. This representation stems from the gradient of whiskers' lengths across the pad, denoting each whisker with a distinct resonance frequency (Hartmann et al., 2003; Neimark et al., 2003; Moore, 2004). Only a specific set of textures will cause each whisker to vibrate in its distinct natural frequency, making each whisker selective for these particular textures. A competing model suggests that surfaces are translated into different kinetic signatures in each vibrissa (Andermann et al., 2004; Hipp et al., 2006; Wolfe et al., 2008). These signatures may be manifested in the velocity of whisker vibration (Shoykhet et al., 2000; Arabzadeh et al., 2003, 2004, 2005, 2006; Jones et al., 2004), spectral composition of whisker vibrations (Hipp et al., 2006), and temporal profiles of whisker motion (Arabzadeh et al., 2006, 2005). The differences between surfaces are expressed by the extent to which different signatures are favored within each vibrissa (Fend et al., 2003; Mehta and Kleinfeld, 2004).

In previous studies, we and others have demonstrated that tactile information is transmitted through high-frequency micromotions, or stick-slip events, superimposed on whisking macromotions (Arabzadeh et al., 2003; Lottem and Azouz, 2008; Ritt et al., 2008; Wolfe et al., 2008; Jadhav et al., 2009). Whereas the rate and magnitude of these events correlate with surface coarse- 
A

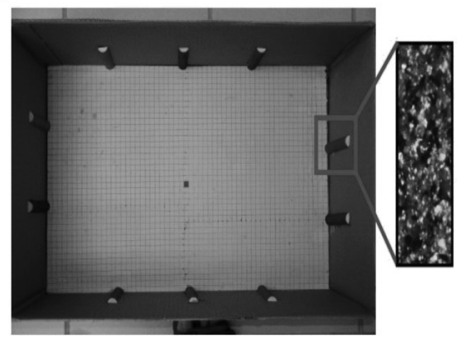

$\mathrm{C}$

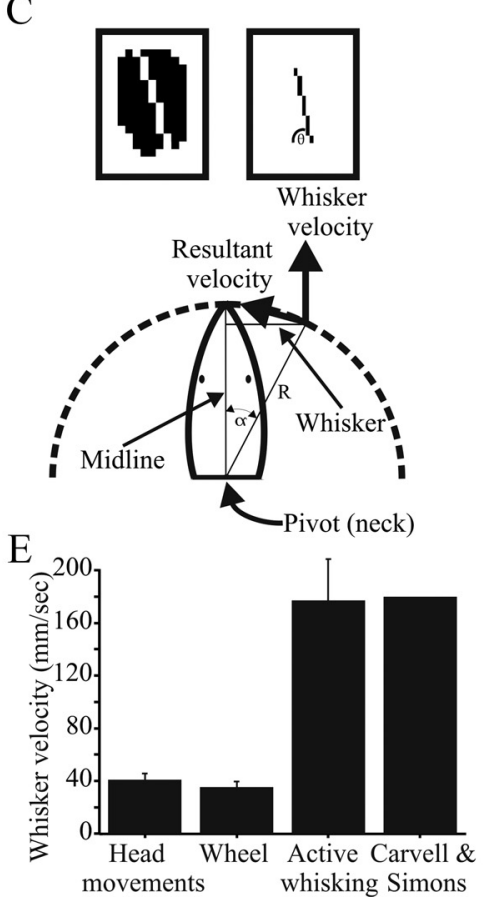

B

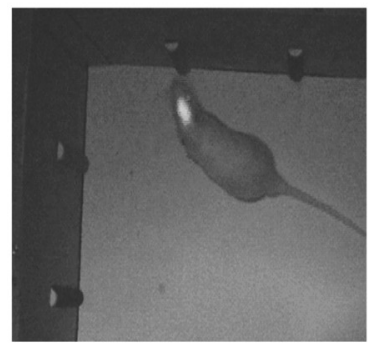

D

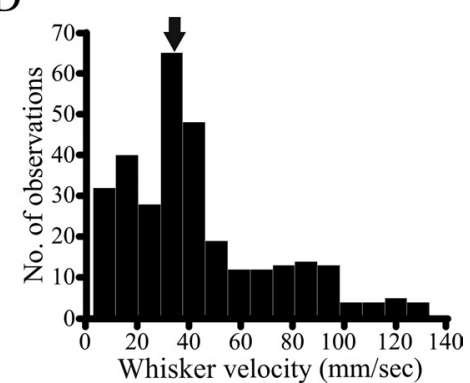

F

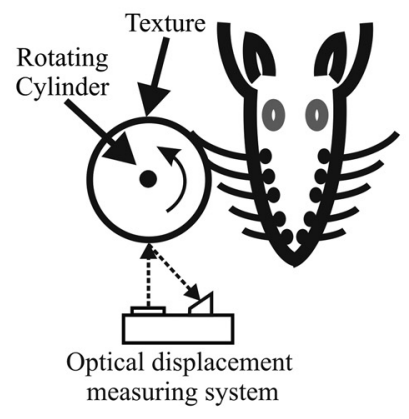

Figure 1. Measurements of head movements in awake behaving rat. $\boldsymbol{A}$, The experimental apparatus, which is composed of a box $(60 \times 45 \mathrm{~cm})$ containing on its sides 10 small objects covered with sandpaper (see inset). $\boldsymbol{B}$, A snapshot of an episode in which the rat approached an object. Notice the elongated lightweight fluorescent device attached to the rat's head. $\boldsymbol{C}$, The top panel shows the cluster of pixels representing the elongated fluorescent device and the line crossing the cluster, representing the head's midline. The bottom panel shows the schematic diagram for calculating whisker motion resulting from head movements (see Materials and Methods). D, Histogram of whisker velocity distribution in a single rat. The arrow indicates the median value. $\boldsymbol{E}$, Comparison of whisker velocity resulting from head movements, rotating cylinder speed, and during active and artificial whisking. $\boldsymbol{F}$, Whisker vibrations were induced by rotation of a texture-covered cylinder. The textures were mounted on a 30-mm-diameter cylinder. The cylinder surface was oriented so that the whisker rested on it and remained in contact during the entire rotation cycle. An optical sensor monitored horizontal motion of the studied whiskers. A noncontact optical displacement measuring system measured the heights of the microfeatures of various surfaces.

ness, the characteristics of whisker resonance vibrations do not vary across textures (Wolfe et al., 2008). These results imply that surface coarseness is encoded not by differential resonant motion across whiskers, but rather by the magnitude and rate of micromotions. The present study explores the mechanisms underlying the translation of surface coarseness into trigeminal ganglion (TG) neuronal discharges during passive whisker movements across textures. We monitored head movements of awake behaving rats while approaching objects. We then replayed these movements in anesthetized rats, monitored whiskers' movements across various surfaces, and concurrently recorded from TG neurons. Our findings suggest that the mechanical and neuronal properties of the system components seem to favor the transmission of small-amplitude high-frequency signals as a mechanism for texture coding.

\section{Materials and Methods}

Recording and stimulation. To quantify whiskers' angular velocity associated with head movements, we placed rats in a box $(n=4$; $60 \times 45 \mathrm{~cm}$ ) containing on its sides 10 small objects covered with sandpaper (Fig. 1A). We monitored their behavior in the dark, using infrared video recording (UI-1225LE-M, IDS Imaging; $752 \times 480,87$ frames per second). By using an elongated lightweight fluorescent device attached to the rats' heads, we were able to monitor their head movements. The posterior end of the device was located near the neck (pivot point for head movements). Episodes in which the rat approached an object were extracted and further analyzed (Fig. $1 B$ ). We then replayed these movements in anesthetized rats, by placing a rotating cylinder covered with textures orthogonal to the whiskers (Fig. 2A). The cylinder was driven by a DC motor (Farnell). Whisker movements were measured under various conditions: whisker movements on sandpaper of four different grades (from coarse-grained to fine-grained; the numbers in parentheses indicate the average grain diameter): P80 (201 $\mu \mathrm{m}), \mathrm{P} 120(125 \mu \mathrm{m}), \mathrm{P} 150$ (100 $\mu \mathrm{m})$, and P220 $(68 \mu \mathrm{m})$. These grades were chosen both in accordance with previous studies (Arabzadeh et al., 2005; Hipp et al., 2006) and based on the findings that rats can discriminate between sandpaper with grain sizes of 400 and $2000 \mu \mathrm{m}$ (Guic-Robles et al., 1989) and between smooth surfaces and rough surfaces having grooves spaced at $90 \mu \mathrm{m}$ intervals (Carvell and Simons, 1990). The textures were mounted on a $30-\mathrm{mm}$-diameter cylinder. The cylinder surface was oriented so that the whisker rested on it and remained in contact during the entire session. The surfaces were placed at $\approx 60$ and $90 \%$ of whisker length. To minimize the number of stimulus combinations and to cover all ranges of whisker types (Neimark et al., 2003; Moore, 2004; Moore and Andermann, 2005), we monitored the movements of the following whiskers arcs: 1, 2, 3, rows B and C. For each texture, we recorded 50 revolutions (trials) per texture of the rotating cylinder, each lasting $\sim 3 \mathrm{~s}$.

Surgical procedures. Adult male Sprague Dawley rats $(n=32 ; 250-350 \mathrm{~g})$ were used. All experiments were conducted in accordance with international and institutional standards for the care and use of animals in research. Surgical anesthesia was induced by urethane ( $1.5 \mathrm{~g} / \mathrm{kg}$ i.p.) and maintained at a constant level by monitoring forepaw withdrawal and corneal reflex, and administering extra doses ( $10 \%$ of original dose) as necessary. Atropine methyl nitrate $(0.3 \mathrm{mg} / \mathrm{kg}$ i.m. $)$ was administered after general anesthesia to prevent respiratory complications. Body temperature was maintained near $37^{\circ} \mathrm{C}$ using a servo-controlled heating blanket (Harvard Apparatus).

After placing subjects in a stereotaxic apparatus (TSE Systems), an opening was made in the skull overlying the TG, and tungsten microelectrodes (2 M $\Omega$, (NanoBio Sensors) were lowered according to known stereotaxic coordinates of TG (1.5-3 mediolateral, 0.5-2.5 anteroposterior) (Shoykhet et al., 2000; Leiser and Moxon, 2006) until units drivable by whisker stimulations were encountered. The recorded signals were amplified (1k), bandpass filtered ( $1 \mathrm{~Hz}-10 \mathrm{kHz})$, digitized $(25 \mathrm{kHz})$, and stored for off-line spike sorting and analysis. The data were then separated to local field potentials (LFP) $(1-150 \mathrm{~Hz})$ and isolated single-unit 
activity $(0.5-10 \mathrm{kHz})$. All neurons could be driven by manual stimulation of one of the whiskers, and all had single-whisker receptive fields. Spike extraction and sorting were accomplished with MClust (by A. D. Redish, available from http://redishlab.neuroscience.umn.edu/MClust/ MClust.html), which is a Matlab (MathWorks) -based spike-sorting software. The extracted and sorted spikes were stored at a $0.1 \mathrm{~ms}$ resolution, and poststimulus time histograms (PSTHs) were computed.

To compare vibrissa velocities to active whisking, we induced artificial whisking (Brown and Waite, 1974; Szwed et al., 2003) by stimulating the buccolabialis motor branch of the facial nerve (Semba and Egger, 1986). The nerve was cut, its distal end mounted on bipolar tungsten electrodes, and was kept moist. Bipolar rectangle electrical pulses $(10-15$ pulses of $100 \mu \mathrm{s}$ at $143 \mathrm{~Hz}$ for $70-105 \mathrm{~ms}$ ) were applied through an isolated pulse stimulator (ISO-Flex; A.M.P.I.) to produce whisker protraction, followed by a passive whisker retraction at $0.5 \mathrm{~Hz}$. The stimulation magnitude was adjusted at the beginning of each recording session to the minimal value that reliably generated the maximal possible movement amplitude (50-200 $\mu \mathrm{A})$. For each texture and free whisking, motor nerve stimulation was delivered at $0.5 \mathrm{~Hz}$ for $200 \mathrm{~s}$, giving a total of 100 whisks per condition. During each experiment, the textures were randomly interleaved. To monitor the drift in whisking and sensor performance, we measured whisker vibrations during free whisking between textures. Whisker displacements transmitted to the receptors in the follicle were measured by an infrared photosensor (resolution, $1 \mu \mathrm{m}$; Panasonic, CNZ1120) placed $2 \mathrm{~mm}$ from the pad. The voltage signals were digitized at $10 \mathrm{kHz}$ and amplified [see Lottem and Azouz (2008) for principles of sensor operation and a description of sensor calibration).

To measure surface coarseness, we used a calibrated noncontact optical displacement measuring system (resolution, $1 \mu \mathrm{m}$; sampled region, $200 \mu \mathrm{m}$; LD1605-2; Micro-Epsilon). We rotated the texturecovered cylinders at velocities corresponding to head movements (see above) and measured the height of surface microfeatures at one point on the texture, over time as the surface rotates (Figs. $1 F, 3 \mathrm{~A}$ ).

Whisker length was measured by plucking the whiskers and measuring their length from tip to tip, using calipers and $3 \times$ magnification under a dissecting microscope.

Data analysis. The whisker movements during passive whisker stimulation and in each sweep across a texture were characterized by their power spectrum, a good representation of whisker kinetics (Arabzadeh et al., 2005; Hipp et al., 2006). Briefly, we used a filtered whisker motion time series recorded during a single trial (bandpass Butterworth type II filter of fourth order: cutoff frequencies $5-500 \mathrm{~Hz}$ ). The Fourier transform of each time series was calculated using the Fast Fourier Transform algorithm in Matlab, and from each Fourier transform, the power spectrum was estimated as the modulus squared of the Fourier transform. The average power spectrum of whisker motion for a single recording session was then an average over all trials performed on that session. Next, we investigated whether some subset of spectral features was particularly relevant to the translation of surface coarseness to neuronal discharges. The approach was to look for the texture-specific differences in the power spectra. The most evident texture-related difference was the power in the signal, i.e., the area below the curves. To look for other

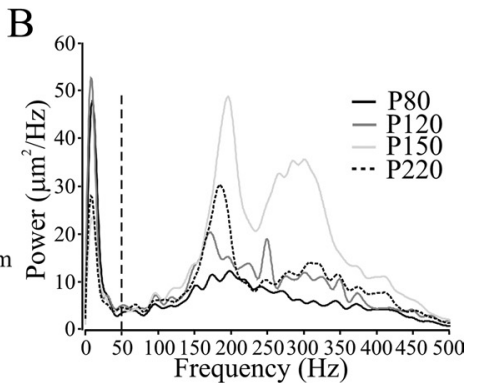

$\mathrm{D}$

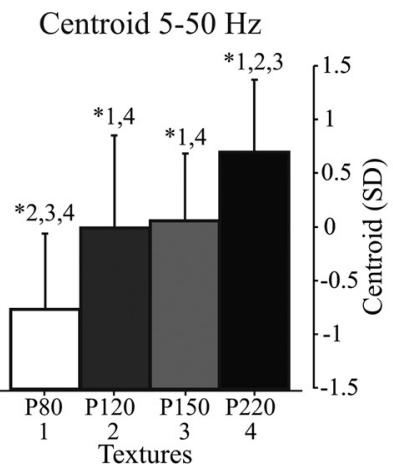

F Centroid $50-500 \mathrm{~Hz}$

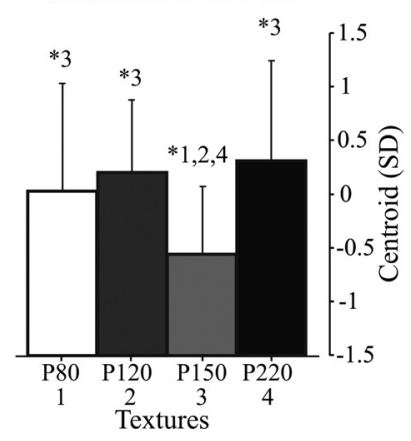

Figure 2. Whisker vibrations are dependent on surface coarseness. $\boldsymbol{A}$, Sample whisker movements associated with texturecovered cylinder rotation for B2 whisker. $\boldsymbol{B}$, Average power spectra of whisker movements across all textures of the whisker shown in $\boldsymbol{A}$. $\mathbf{C}-\boldsymbol{F}$, Dependence of power $5-50 \mathrm{~Hz}$, centroid $5-50 \mathrm{~Hz}$, and power $50-500 \mathrm{~Hz}$, centroid $50-500 \mathrm{~Hz}$ on surface coarseness. Error bars in the figure indicate the SE. ${ }^{*} p<0.01$, significantly different from the indicated group.

potentially informative features, we then normalized the area under the curve of all the spectra, thereby excluding power as a feature. After this normalization, we used the "centroid" - a measure related to the center of gravity within the power spectrum (Hipp et al., 2006).

$\omega_{1}, \omega_{2}, \ldots, \omega_{\mathrm{n}}-$ frequencies

$F(\omega)$ - Fourier transform of the signal

$$
\text { Power }=\sum_{i=x}^{y}\left|F\left(\omega_{i}\right)\right|^{2},
$$

where $x$ and $y$ are the range of indices: $\omega_{x 1}=5, \omega_{y 1}=50, \omega_{x 2}=50, \omega_{y 2}=500$;

$$
\text { Centroid }=\frac{\sum_{i=x}^{y} \omega_{i} * F\left(\omega_{i}\right)}{\sum_{i=x}^{y} F\left(\omega_{i}\right)} .
$$

The transfer function from surface distance profiles and whisker vibrations is modeled by the linear, time-invariant function.

$x=$ input signal vector, $y=$ output signal vector.

The transfer function is $T_{x y}$, the quotient of the cross-power spectral density $\left(P_{x y}\right)$ of $x$ and $y$ and the power spectral density $\left(P_{x x}\right)$ of $x$, where $x$ is the texture signal and $y$ is the whisker signal (period duration, $1 \mathrm{~s}$; 
A

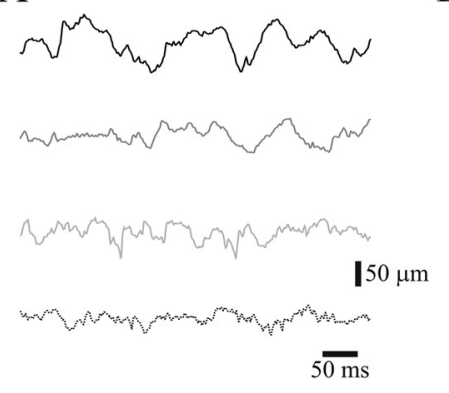

$\mathrm{C}$

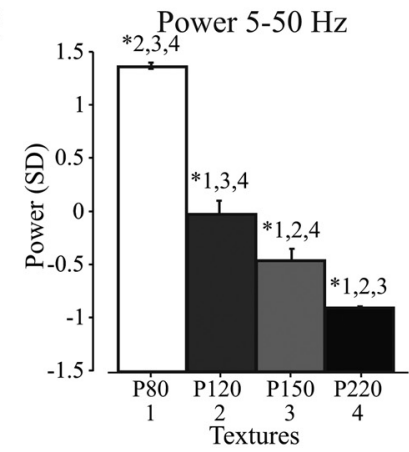

$\mathrm{E}$

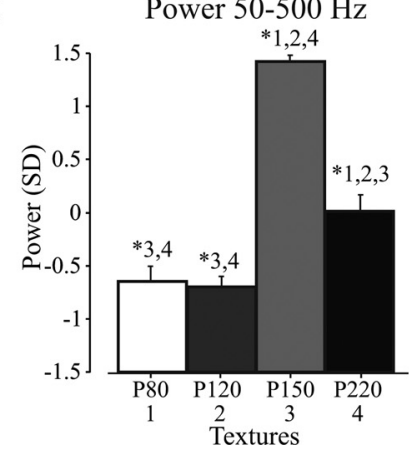

$\mathrm{B}$

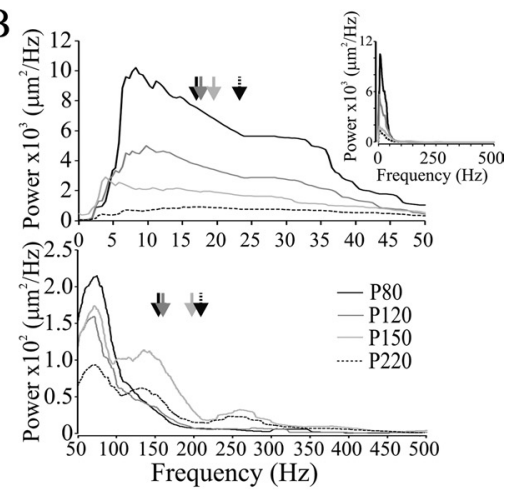

$\mathrm{D}$

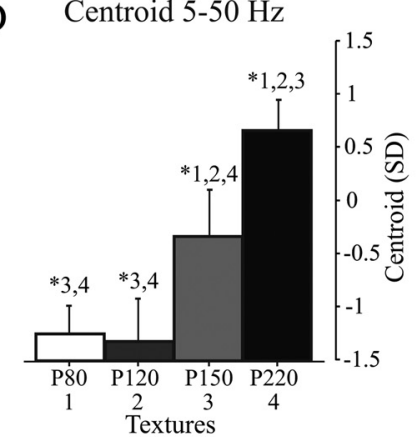

F Centroid 50-500 Hz

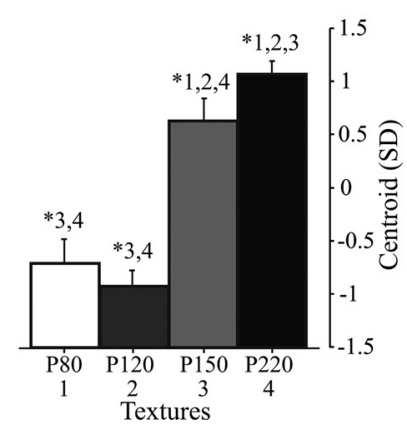

Figure 3. Characterization of surface coarseness. $\boldsymbol{A}$, Sample distance profile associated with different texture. $\boldsymbol{B}$, Average power spectra of distance profiles across all textures of the textures shown in $A$. The spectra were split into two frequency ranges. The inset shows the full range. $C-F$, Dependence of power $5-50 \mathrm{~Hz}$, centroid $5-50 \mathrm{~Hz}$, and power $50-500 \mathrm{~Hz}$, centroid $50-500 \mathrm{~Hz}$ on the different texture grades. Error bars in the figure indicate the $\mathrm{SE} .{ }^{*} p<0.01$, significantly different from the indicated group.

sampling frequency, $2 \mathrm{kHz}$ ). The power spectral density was calculated using Welch's averaged, modified periodogram method of spectral estimation (Oppenheim and Schafer, 1999).

$$
T_{x y}(f)=\frac{P_{x y}(f)}{P_{x x}(f)} .
$$

Power spectra of whisker motion were calculated using a single-tapered time-frequency spectrum, calculated with a time window of $100 \mathrm{~ms}$ and step size of $1 \mathrm{~ms}$.

To measure whisker natural frequency (NF), we used the impulse method in which a small object was placed on the rotating cylinder. An impulse was delivered through cylinder rotation to each whisker, and the resulting decaying oscillation was measured with the infrared photosensor (see Fig. 6A). Quantification of the NF was done across multiple repetitions by calculating the power spectrum (see Fig. $6 B$ ). We then calculated the SD of the spectral power (dashed horizontal line). Each whisker was assigned a range of natural frequencies which were above 1 SD.

Whisker angle at the base (the most proximal point of the tracked whisker) was computed: Angle at base $=\arctan$ (measured whisker trajectory/measuring distance) measuring distance $\approx 2 \mathrm{~mm}$.
In the experiments of awake animals, we quantified the angular velocity associated with head movements by computing the angular position of a whisker at a distance of $10 \mathrm{~mm}$ from the pad for each frame of the movie (Fig. $1 C ; 33.7 \mathrm{~ms})$. The patch was transformed into a cluster of pixels $(720 \times 480$ pixels $)$ in the $X-Y$ plane. Linear regression was used to find the line crossing the cluster, representing the head's midline.

The angular position of the head in respect to the neck (Pivot) was defined as $-\operatorname{Arctan}(a)$, where $a$ is the slope of the linear regression. We assume that the head movement is expressed mainly in slope but not in the intercept, yaw, and pitch. "Angular velocity" is the derivative of angular positions, and represents the angular velocity of the head's midline around a pivot (the neck).

"Whisker velocity" is the velocity of a point on a whisker ( $1 \mathrm{~cm}$ lateral to the midline) (Fig. $1 C$ ) in parallel to the head's midline, and is defined:

$$
V=\omega * R * \sin \alpha .
$$

$V$ is whisker velocity, $\omega$ is angular velocity, $R$ is distance between the pivot and the whisker, and $\alpha$ is the angle between the midline and $R$. Since $R^{\star} \sin \alpha=1 \mathrm{~cm}$, then

$$
V=\omega \text {. }
$$

To deal with large ranges of neuronal firing rate and whisker vibrations in different animals, we used the standard score, which is a dimensionless quantity derived by subtracting the population mean from an individual raw score and then dividing the difference by the population SD. This normalization was done on data from the same whisker across different textures; i.e., population mean was calculated for each whisker or neuron across different textures.

$$
z=\frac{x-\mu}{\sigma} .
$$

where $x$ is a raw score to be standardized, $\sigma$ is the $\mathrm{SD}$ of the population, and $\mu$ is the mean of the population.

The significance of the differences between the measured parameters was evaluated using one-way ANOVA. When significant differences were indicated in the $F$ ratio test $(p<0.05)$, the Tukey method for multiple comparisons was used to determine those pairs of measured parameters that differed significantly within the pair $(p<0.05$ or $p<0.01)$. Averaged data are expressed as mean $\pm \mathrm{SE}$. Error bars in all the figures indicate the SE.

\section{Results}

To examine whether tactile information may be transmitted through passive whisker movements, we placed rats in a box $(n=4 ; 60 \times 45 \mathrm{~cm})$ on whose sides we placed 10 small objects covered with sandpaper of different grades of coarseness (Fig. $1 A, B)$. We monitored their behavior while approaching various objects in the dark, using infrared video recording. We quantified whisker velocity associated with head movements by computing the angular position of the head relative to the neck for each frame of the movie (see Materials and Methods) (Fig. 1C). 
The results of these calculations show that whisker velocities were broadly distributed $(n=4)$ (Fig. $1 D$; median, $40.9 \pm$ $4.7 \mathrm{~mm} / \mathrm{s})$. These velocities were lower than whisker velocities during active whisking (Fig. $1 \mathrm{E}$; Median, $177.3 \pm 31.1$ $\mathrm{mm} / \mathrm{s}$ ) (Lottem and Azouz, 2008) and those reported in previous studies (180 $\mathrm{mm} / \mathrm{s}$ ) (Carvell and Simons, 1990; Ritt et al., 2008).

To examine how surface coarseness is transformed into vibration signals in whiskers, and how these vibrations are expressed in neuronal discharges, we replayed these passive whisker movements across different surfaces by covering the face of a rotating cylinder with several grades of sandpaper with different coarseness. The cylinder face was placed orthogonally to the whiskers, so that the whisker rested on it (Fig. $1 F$ ). These surfaces were placed at whisker lengths of $\approx 60 \%$ and $\approx 90 \%$. Whisker movements were measured in response to sandpaper of four different grades: P80, P120, P150, and $\mathrm{P} 220$. The angular velocity was controlled using a DC motor driven at $38 \pm 5 \mathrm{~mm} / \mathrm{s}$

(Fig. $1 E$ ) to replicate head velocity. We next examined which aspects of whisker vibrations are dependent on surface coarseness, by computing the power spectrum of the B2 whisker's raw vibrations (Fig. 2A,B). The corresponding power spectra, as shown in Figure $2 B$, can be broken down into two major components: lower power signals at frequencies $5-50 \mathrm{~Hz}$ and the large power signals at frequencies $50-500 \mathrm{~Hz}$ (vertical dashed line). This separation was found to be typical of all whiskers studied $(n=32)$.

Figure $2 B$ indicates that the difference between surfaces may be expressed by their power spectrum characteristics. To quantify these differences, we separated the signal, as noted above, into its low and high frequencies (bandpass Butterworth type II filter of fourth order: cutoff frequencies $5-50 \mathrm{~Hz}$ and cutoff frequencies $50-500 \mathrm{~Hz}$ ). We then calculated the integrated power of whisker vibrations in the ranges of $5-50 \mathrm{~Hz}(5-50 \mathrm{~Hz}$ power) and $50-500$ $\mathrm{Hz}(50-500 \mathrm{~Hz}$ power) as well as the centroids (5-50 Hz centroid, $50-500 \mathrm{~Hz}$ centroid; for further details, see Materials and Methods). The dependence of these parameters on surface coarseness across whisker $\operatorname{arcs} 2$ and $3(n=22)$ is shown in Figure 2, $C-F$. Reduction in surface coarseness $(\mathrm{P} 80 \rightarrow \mathrm{P} 220)$ resulted in an overall reduction of the $5-50 \mathrm{~Hz}$ power and in an upward shift of the corresponding centroid (Fig.2C,D). Surface coarseness had only minor effects on $50-500 \mathrm{~Hz}$ centroid, while increasing initially and finally decreasing the $50-500 \mathrm{~Hz}$ power (Fig. $2 E, F$ ). These results indicate that surface coarseness is expressed in an approximately linear manner by the characteristics of whisker vibrations at lower frequencies, whereas at higher frequencies its expression by these characteristics seems to be more complex.

Given that sandpaper coarseness is defined by its average grain diameter, and that the sandpaper-covered rotating surfaces were placed perpendicular to the whiskers, the average grain diameter of various sandpaper grades may be expressed in diverse radial distance profiles. To examine these profiles we used a calibrated noncontact optical displacement measuring system (Fig. $1 F$ ). Measurements of the heights of microfeatures of various surfaces, over time, as each surface rotates (see Materials and Methods) are shown in Figure $3 A$. We then computed the power spectrum of these height signals (Fig. $3 B$ ). The average power spectrum of all surfaces (each surface was sampled at several locations) is shown in Figure $3 B$ (inset). To relate the changes in the surfaces to modulation in whisker vibrations, we also split the scan power spectrum into the same two components: signals at frequencies 5-50 $\mathrm{Hz}$ (Fig. 3B, top) and signals at frequencies $50-500 \mathrm{~Hz}$ (Fig. $3 B$, bottom). The figure indicates that the differences between surfaces may be expressed in their power spectrum characteristics. To quantify these differences, we calculated the aforementioned power and centroid parameters for all surfaces. The dependence of these parameters on surface coarseness is shown in Figure 3, $C-F$. Reducing surface coarseness resulted in an overall reduction of the $5-50 \mathrm{~Hz}$ power and an upward shift of the corresponding centroid. Surface coarseness reduction led to an upward shift of the $50-500 \mathrm{~Hz}$ centroid, whereas its effects on $50-500 \mathrm{~Hz}$ power were similar to those of surface coarseness $50-500 \mathrm{~Hz}$ power in whisker vibrations (compare Fig. $3 E$ with $2 E$ ). These results suggest that surface coarseness as defined by the standard industrial Grit measure may not be the same as the measured coarseness as characterized by the more refined heights profiles.

To determine which of these four surface coarseness characteristics is translated into whisker vibrations, we correlated our scan variables with those obtained for all whiskers $(n=32)$. Linear regression was then used to evaluate the parameters of each distribution. We found that surface coarseness and whisker vibrations were mainly correlated through the $50-500 \mathrm{~Hz}$ power variable (Fig. $4 C$ ) and, to a lesser degree, through $5-50 \mathrm{~Hz}$ power and centroid (Fig. $4 A, B$ ), suggesting again that surface coarseness information is carried mainly by high-frequency whisker vibrations (Fig. $2 B$ ). No correlation was found between texture and whisker $50-500 \mathrm{~Hz}$ centroid variable. Close examination of the unimodal shape of the surfaces' power spectra (Fig. 3B) and the corresponding bimodal profile of whisker vibration spectra (Fig. 2B) suggests that whiskers serve as filters in which lower 
A

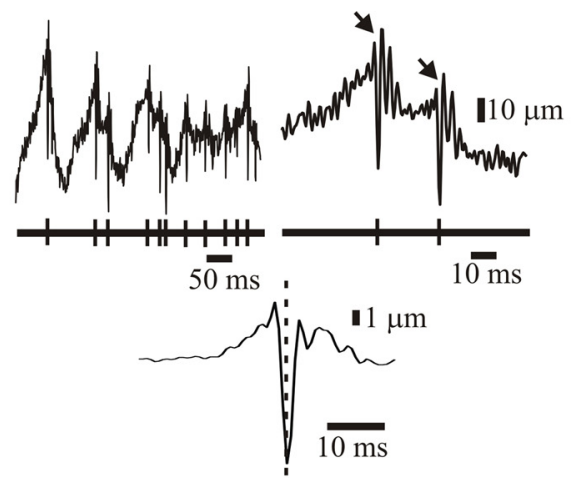

$\mathrm{B}$

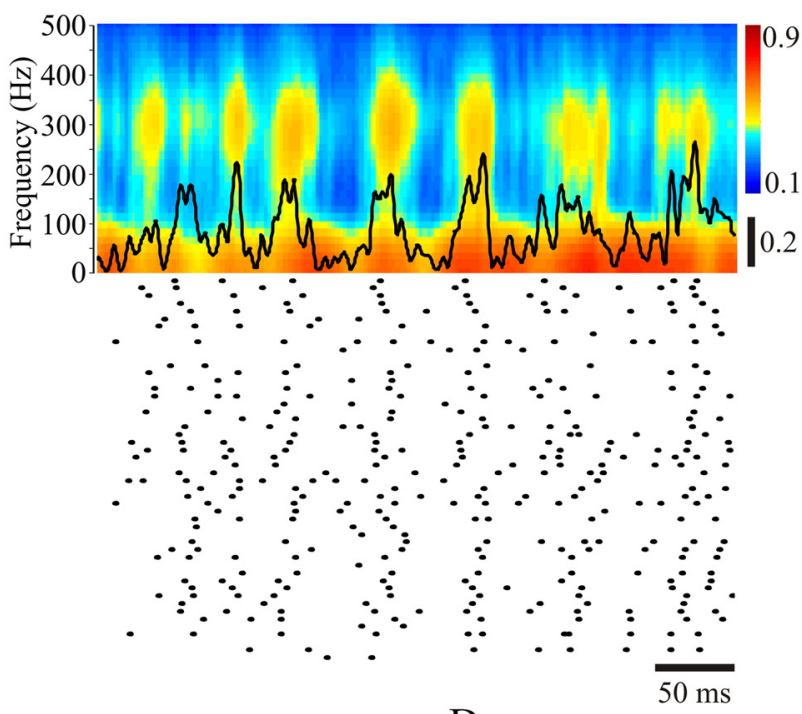

$\mathrm{C}$
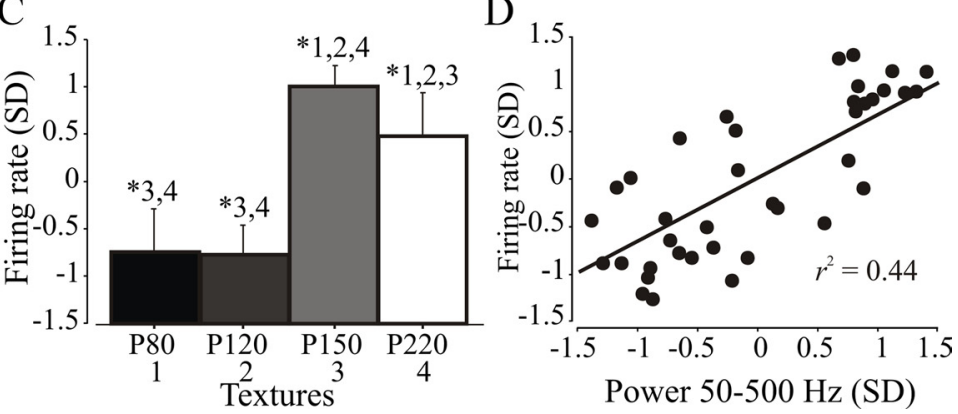

Figure 5. Neuronal firing rates are highly dependent on power $50-500 \mathrm{~Hz}$ in whisker vibrations. $\boldsymbol{A}$, Sample whisker movements associated with texture-covered cylinder rotation for P150 surface (top). The traces on the right show a segment from the left at a higher magnification. The middle panels show the corresponding raster plot of a neuron responding to cylinder rotation. The bottom shows the STA of whisker movements. $\boldsymbol{B}$, Spectrogram of whisker movements and the corresponding PSTH and raster plot of the neuron in $\boldsymbol{A}$. C, Dependence of neuronal firing rates on surface coarseness. D, Correlation between power $50-500 \mathrm{~Hz}$ in whisker vibration signal and neuronal firing rates. Error bars in the figure indicate the $S E$. ${ }^{*} p<0.01$, significantly different from the indicated group.

frequencies are more attenuated than higher frequencies. To examine these filtering properties, we calculated the transfer function between these two signals (see Materials and Methods) and found that, indeed, higher-frequency components are less attenuated (Fig. 4D). Moreover, we did not find any difference between the transfer functions for the various textures. This behavior was found to be characteristic of whiskers from all arcs (see Fig. 7; $n=32$ ). Together, these results suggest that whiskers transmit the high-frequency components of the various surfaces' radial height profiles, and that this property is texture independent.

To examine the relationship between whisker vibrations and neuronal discharges, we recorded extracellularly from 32 TG neurons while monitoring whisker movements across surfaces. An example of the relationship found is shown in Figure $5 A$ for a single neuron responding to $\mathrm{B} 2$ whisker stimulation. The upper columns depict whisker vibrations and neuronal discharges during surface (P150) contact at two temporal magnifications. These panels show that many of the spikes appear to arise from rapid whisker vibrations (Fig. 5, slanted arrows) (Lottem and Azouz, 2008). This observation is confirmed by the spike-triggered average (STA) of whisker trajectory over a \pm 100 ms time lag for all spikes in a session, shown in the lower panel. It reveals that action potentials in TG neurons commonly arise from high-frequency micromotions (Lottem and Azouz, 2008, their Fig. $2 B$, lower panel).

To further characterize this relationship, we computed the spectrogram of whisker vibration signals during multiple rotations of the texture-covered cylinder and correlated it with discharge rates of TG neurons. An example of this relationship is shown for a B3 neuron responding to a P80 surface (Fig. $5 B$ ). The raster plot and the PSTH of the neuron, shown in black, indicate a high degree of correlation between discharge rate and highfrequency whisker vibrations. This behavior was found to be typical of all neurons in the study $(n=32)$. Finally, to examine whether information about surface coarseness is translated into neuronal activity, we calculated the discharge rates of all neurons across all whiskers in response to the various surfaces. The results of these calculations are shown in Figure 5C. The panel shows that neuronal discharge rate varies as a function of surface coarseness in a complex manner. In the current study, we used the following surfaces: P80, P120, P150, and P220. The first two surfaces are outside the range of previous studies (von Heimendahl et al., 2007; Wolfe et al., 2008; Jadhav et al., 2009). The latter two surfaces are within the range of the literature and show that coarser surfaces generate higher firing rates than do smoother surfaces (Fig. 5C). In view of Figures $2 E$ and $3 E$, as well as the current figure, these results suggest that TG neurons transmit mainly high-frequency components in surface distance profiles and whisker vibrations.

To further support this conclusion, we determined which of the aforementioned whisker vibration characteristics is translated into neuronal firing. We correlated these variables with discharge rates in a subset of neurons $(n=22$; neurons that fire above an arbitrarily chosen threshold of $1 \mathrm{~Hz}$ ). Linear regression was used to evaluate the parameters of each distribution. We found that, out of four parameters, neuronal firing rates were only correlated to the $50-500 \mathrm{~Hz}$ power variable (Fig. $5 D ; r^{2}=$ 
$0.44)$ and (to a lesser extent) to the 5-50 $\mathrm{Hz}$ centroid $\left(r^{2}=0.09\right)$. Together, these results suggest that surface coarseness information is carried mainly by highfrequency whisker vibrations. These are transmitted to TG neurons and are expressed by their discharge rate. Thus, head and body movements may serve as an effective means of acquiring tactile information.

We tested for whisker resonance and its effects on neuronal discharge by examining the relationship between the frequency spectrum of whisker vibrations during contact with objects and the intrinsic resonance frequencies of the whiskers. The NF for each whisker was measured by delivering an impulse to the whisker, and the frequency from the resulting decaying oscillations was calculated (see Materials and Methods). An average response for a $\mathrm{B} 3$ whisker to 50 repetitions of object contact is shown in Figure 6 A. Quantification of the NF was done across multiple repetitions by calculating the power spectrum. Each whisker was assigned a range of natural frequencies (the frequencies that crossed the threshold of 1 SD; gray area125-178 Hz). Whisker vibrations in response to object contact could be broadly classified into three types of events: sticks (events in which speed decreases gradually, corresponding to the whisker being stuck to the object and moving with it), slips (events in which whisker speed suddenly increased, corresponding to whisker release), followed by transient high-frequency ringing (Fig. 6A, events 1-5). To determine the temporal relations between these events and neuronal discharges, we calculated for each neuron the PSTH and the number of spikes in each event. The PSTH for the neuron in Figure $6 C$ shows that most spikes occur during the slip event. This is further confirmed in Figure $6 D$, showing that all neurons discharge mainly during slips and hardly ever during the following transient decaying ringing.

To characterize neuronal behavior during surface contact, we calculated the STA of whisker vibrations (Fig. 7A). Observation of the STA for all spikes reveals that spikes mostly arise from high-frequency micromotions (slip events; see above). Whisker vibrations around spike generation, during surface contact, could also be broadly classified into the three types of events (see above). The amplitude of slip events that resulted in spike discharge did not depend on surface coarseness and was not related to neuronal discharge rate (see supplemental Material: Part 1 and correspond-
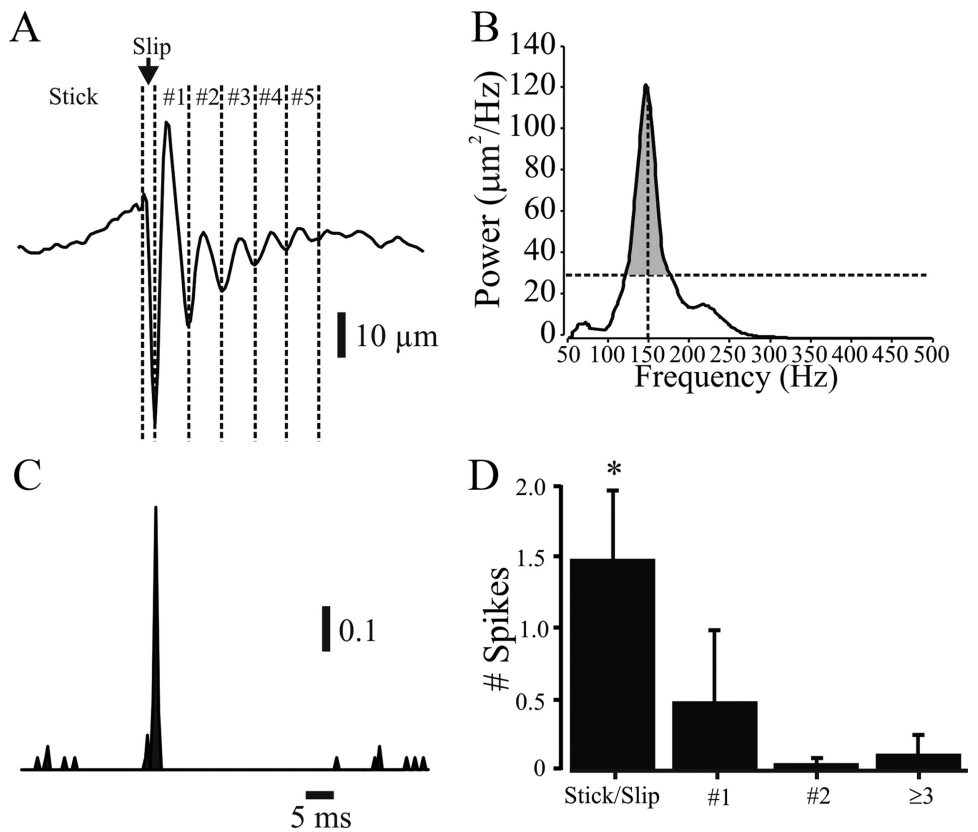

Figure 6. Neuronal discharges are associated with stick and slips events during impulse response. $\boldsymbol{A}$, Impulse method for measuring NF. The responses are divided into several events: stick, slip, \#1-5-multiple phases of resonant ringing. $\boldsymbol{B}, \mathrm{NF}$ is calculated from the peak in the power spectrum. For each whisker we assigned a range of NF frequencies, which crossed the threshold ( $>1$ SD of the power spectrum). C, PSTH of the neuron responding to the whisker vibrations in $\boldsymbol{A}$. Neuronal firing is associated mainly with slip events. $\boldsymbol{D}$, Histogram of neuronal discharge during the different events in a subset of the neurons $(n=$ 11). Error bars in the figure indicate the SE. ${ }^{*} p<0.01$, significantly different.
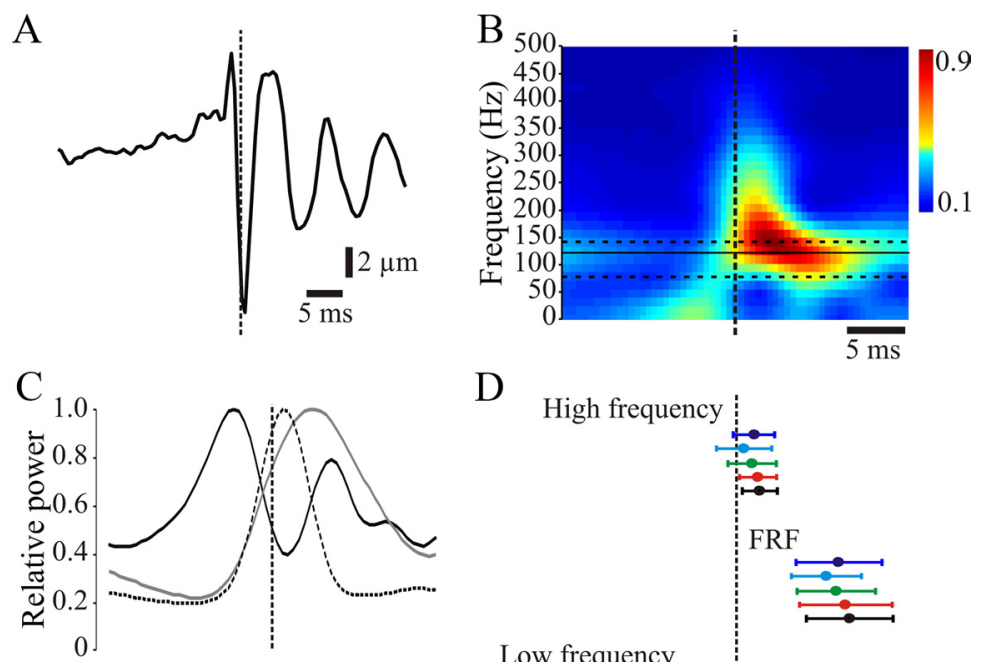

D High frequency
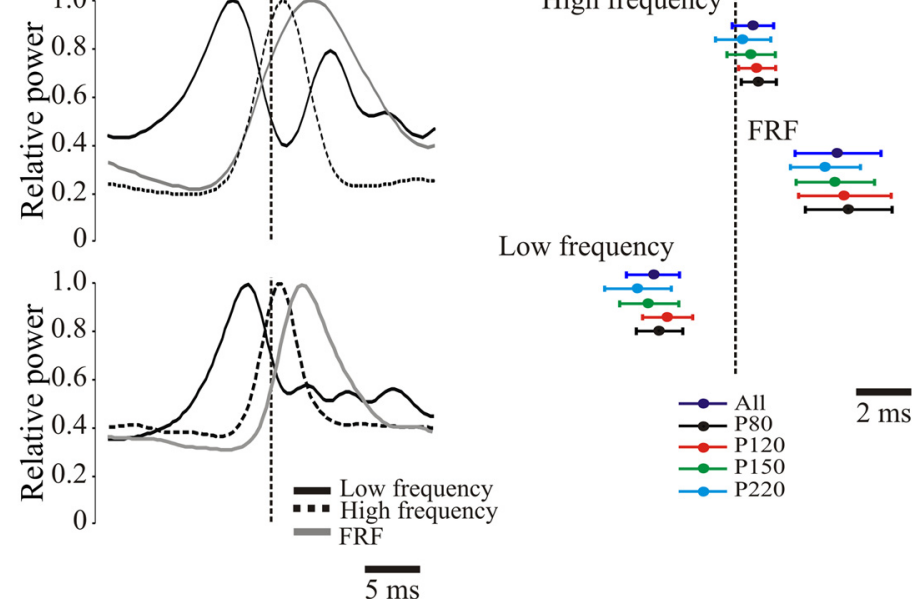

Figure 7. Neuronal discharges are associated with stick and slip events during surface contact. $\boldsymbol{A}$, An example of STA of whisker movements. Neuronal firing (dashed vertical line) is associated with stick and slip events followed by ringing of the whisker. $\boldsymbol{B}$, Average spectrogram of whisker movements for all spikes showing lower frequencies preceding the spikes and high frequencies during and after the spikes. Dashed and filled horizontal lines indicate the NF and its lower and upper bound. $\boldsymbol{C}$, Temporal profiles of the neuron $\boldsymbol{A}$ (top) and all neurons (bottom) of the power at the NF range (gray line), frequencies lower than the threshold (black line), and frequencies higher than the threshold (dashed line). D, Position of the peaks of the three frequency bands relative to spike time for all neurons and all textures. Error bars in the figure indicate the SE. 
A

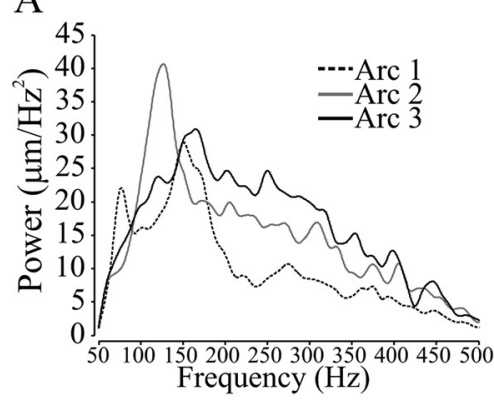

C

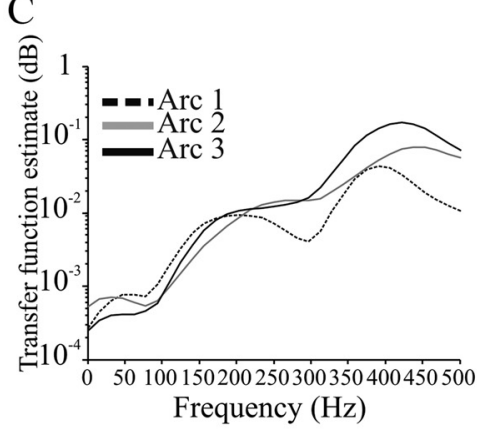

$\mathrm{E}$

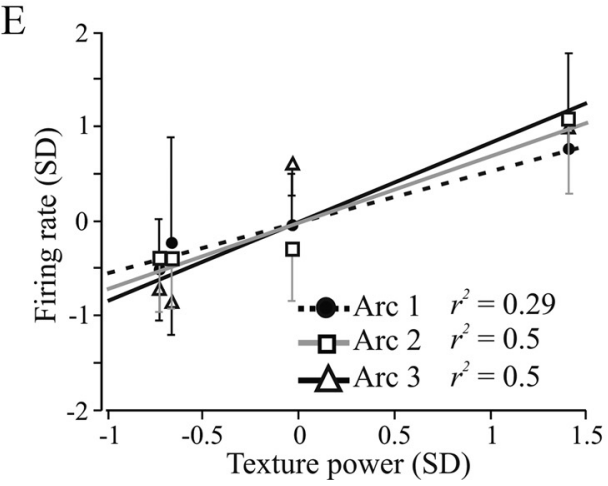

Figure 8. Whisker length influences the transmission of tactile information. $A$, Average power spectra of whisker movements in different arcs across all surfaces. $\boldsymbol{B}-\boldsymbol{D}$, Dependence of whisker vibration power $50-500 \mathrm{~Hz}$, transfer function, and firing rates on whisker length. $\boldsymbol{E}$, Correlation between power $50-500 \mathrm{~Hz}$ in the distance profiles of different whiskers and neuronal firing rate. Error bars in the figure indicate the SE. ${ }^{*} p<0.01$, significantly different from the indicated group.

ing Fig. S1, available at www.jneurosci.org as supplemental material). Because responses of neurons in various stages of the whisker somatosensory system better reflect whisker deflection velocity than amplitude (Shoykhet et al., 2000; Krupa et al., 2001; Arabzadeh et al., 2004; Jones et al., 2004; Stüttgen et al., 2006), we also calculated the velocity profile of slip events and find again that the magnitude of slip velocity that resulted in spike discharge did not depend on surface coarseness (see supplemental Material: Part 1 and corresponding Fig. S1, available at www. jneurosci.org as supplemental material). We then calculated the spectrogram of whisker vibrations for each spike (to maintain the temporal phase of these events to spike generation). The average spectrogram of whisker movements for all spikes reveals lower frequencies before spikes (stick), higher frequencies during spikes (slip), and postspike ringing of the whisker, approximately at its NF (Fig. 7B). To quantify this behavior, we calculated the power at the NF range for this neuron $(77-144 \mathrm{~Hz}$; see above), at frequencies lower than the threshold (corresponding to whisker stick, Fig. $7 B$, beneath the lower dashed line, 5-76 Hz), and at frequencies higher than the threshold (corresponding to whisker slip, (Fig. 7B, above the upper dashed line, $144-500 \mathrm{~Hz}$ ) in $40 \mathrm{~ms}$ epochs centered on spikes. The results (Fig. 7C, top) show that peak power for each of these three frequency bands appears at different times relative to the spikes. These results were consistent in a subset of neurons [Fig. 7C, bottom; neurons that discharge $>1 \mathrm{~Hz}$ and having a distinct NF (see Materials and Methods); $n=11 ; \operatorname{arc} 1=3 ; \operatorname{arc} 2=5 ; \operatorname{arc} 3=3$ ]. We calculated the peak power for each of these frequency bands. The average peak for each of the bands, across different textures, relative to spike time, is shown in Figure $7 D$. The panel shows that spikes are preceded by low frequencies (stick), take place mostly during high frequencies (slip), and are followed by ringing at the NF. Thus, the three bands seem to be well separated temporally, relative to spikes (Fig. 7D). The accumulative power in the natural frequencies range did not depend on surface coarseness and was not related to neuronal discharge rate (see supplemental Material: Part 2 and corresponding Fig. S2, available at www.jneurosci.org as supplemental material). Together, these results indicate that under our experimental setup, resonance vibrations in whiskers during surface contact primarily represent transient ringing after discrete high-velocity movement events that cause neurons to discharge.

It has been suggested that the mechanical properties of whiskers play a critical role in the translation of surface coarseness to whisker vibrations (Andermann et al., 2004). Therefore, we examined the effects of these mechanical properties on the translation of surface coarseness into whisker vibration signal and neuronal discharge rates. Initially, we measured whisker length and NF as a function of arc position and found an anterior-posterior gradient, in agreement with previous reports (Brecht et al., 1997; Hartmann et al., 2003; Neimark et al., 2003; Ritt et al., 2008; Wolfe et al., 2008) ( $\operatorname{arc} 1$, $n=3$; length $=3.9 \pm 0.6 \mathrm{~cm}, \mathrm{NF}=108.9 \pm 19.5 \mathrm{~Hz}$; $\operatorname{arc} 2, n=$ 5; length $=3.0 \pm 0.4 \mathrm{~cm}, \mathrm{NF}=118 \pm 6.1 \mathrm{~Hz}$; $\operatorname{arc} 3, n=3$; length $=2.1 \pm 0.4 \mathrm{~cm}, \mathrm{NF}=172 \pm 17.1 \mathrm{~Hz}$ ). We then compared the effects of various sandpaper types on power spectra for these three arcs. The results are shown in Figure $8 A$ for all whiskers presented with a P150 texture $(n=32)$. This panel shows that, for the same texture, the spectral contents varies across arcs. This observation is confirmed by calculating the $50-500 \mathrm{~Hz}$ power in the various arcs (Fig. $8 \mathrm{~B}$ ); the results show that longer, thicker whiskers transmit less power in the high-frequency range.

We have shown that whiskers serve as filters in which lower frequencies are attenuated more than higher frequencies (Fig. 4). To examine the influence of the biomechanical properties of whiskers on this transformation, we calculated the transfer function between surface distance profile and whisker signal (see Materials and Methods) in different arcs. Figure $8 C$ shows that shorter, slender whiskers transmit more high-frequency information. Given that neuronal discharge rate is highly correlated to 
$50-500 \mathrm{~Hz}$ power (Fig. 5), we expected differences across arcs to be expressed in neuronal discharge rates, and found that the percentage of neurons that fire below an arbitrarily chosen threshold $(1 \mathrm{~Hz})$ is highest in arc 1 (arc 1, 50\%; $\operatorname{arc} 2,25 \%$; $\operatorname{arc} 3,20 \%)$. Moreover, the neurons that cross the discharge rate threshold in arc 1 fire at lower rates than do neurons in arcs 2 and 3 (Fig. 8D). Finally, the resonance theory suggests that each whisker is preferentially excited by a specific set of textures. To test this prediction we compared the relations of the whiskers in different arcs to a range of different textures. We found that none of the whiskers displays texture selectivity (Fig. $8 E$ ), again consistent with constant bandpass filter. These findings indicate that the mechanical properties of whiskers influence the translation of surface coarseness into neuronal activity, suggesting that the various whiskers may serve in different roles in the transmission of tactile information.

To determine the influence of surface distance on texture signals, the proximal edge of the surface was placed at $\approx 60 \%$ and $\approx 90 \%$ of whisker length (Szwed et al., 2003). An example of this effect for a single neuron responding to the $\mathrm{C} 2$ whisker is shown in Figure 9A. The upper and lower rows depict whisker vibrations and neuronal discharges during surface (P150) contact at these two distances. This example shows that surface distance has a critical influence on whisker vibrations and consequently on neuronal discharge rate. To quantify the effect we plotted the normalized firing rates (Fig. 9B) for a subset of textures and neurons $(n=4)$ at the two distances. These two panels show that at a distant object location, the differences between the surfaces are more pronounced. Finally, to examine the absolute differences between the two distances we compared neuronal discharge rates. The panel in Figure $9 C$ shows that TG neurons discharge less when objects are closer. These results suggest that object distance adds complexity to the overall picture: whereas discharge rates carry information about object distance, object distances do influence the discrimination of surface coarseness.

To compare the translation processes during passive and active whisker contact with surfaces, we induced artificial whisking in anesthetized rats and monitored the movements of whiskers across surfaces. Artificial whisking was induced by electrically stimulating cranial nerve 7 , generating $6 \mathrm{~Hz}$ whisking movements (Brown and Waite, 1974; Szwed et al., 2003) that resemble whisker trajectories in awake rats (Berg and Kleinfeld, 2003). Whisker movements across surfaces resulted in texture-related highfrequency micromotions riding on whisking macromotion signals (Lottem and Azouz, 2008) (Fig. 10 A, top). To separate them, we used bandpass filters (see above), which produced the traces shown in panels 1 and 2 of Figure $10 \mathrm{~A}$. To characterize the dependence of whisker vibrations on surface coarseness during active whisking, we computed the power spectrum of the highfrequency micromotions (example in Fig. 10B). This example shows that the surface differences are expressed in the power spectrum. We then correlated the integrated power of surface distance profiles with whisker vibrations. The results are shown in Figure 10C. We found that during active whisking, surface coarseness and whisker vibrations were mainly correlated through the $50-500 \mathrm{~Hz}$ power variable. This is further verified by calculating the transfer function between surface distance profile and whisker signal during active whisking for various surfaces. Figure $10 \mathrm{D}$ shows again that surface coarseness information is carried mainly by high-frequency whisker vibrations. Our conclusion is that the process by which tactile information becomes available to the animal may be a similar one during both passive and active whisker movements.
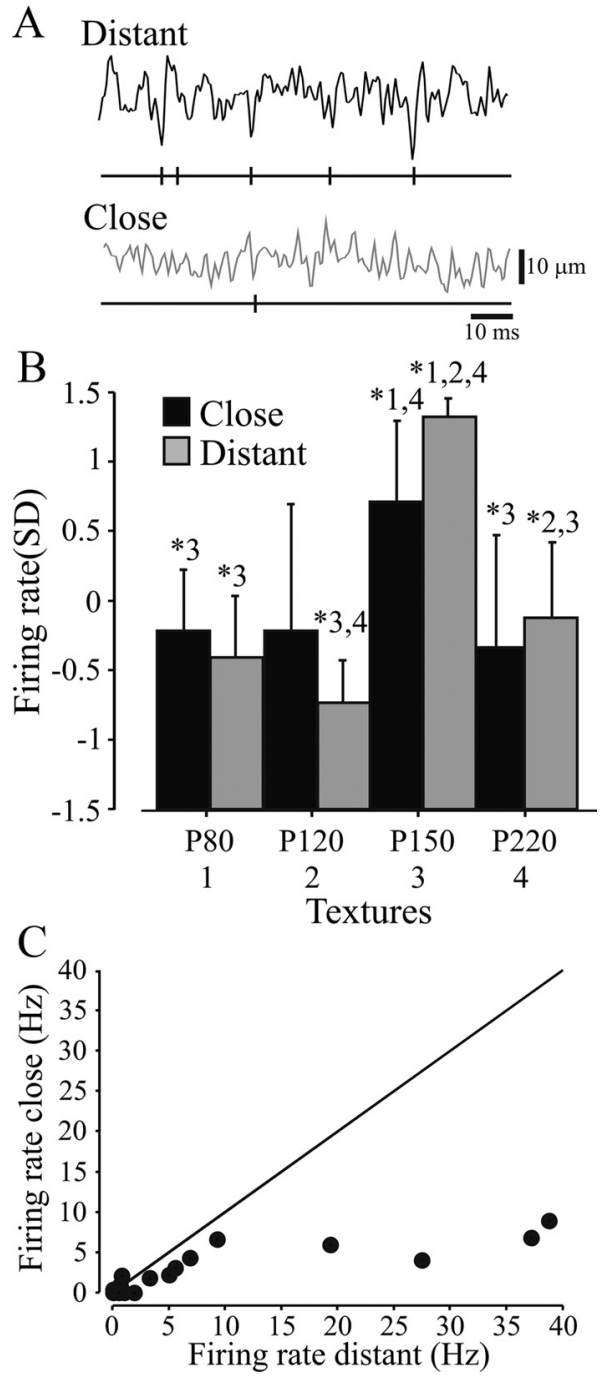

Figure 9. Neuronal firing rates are highly dependent on surface distance. $\boldsymbol{A}$, Sample C2 neuron and whisker movements associated with texture-covered cylinder rotation for P150 surface at two distances (top). The bottom shows the corresponding spike trains of a neuron responding to cylinder rotation. $B, C$, Comparison of neuronal firing rates at the two distances. Error bars in the figure indicate the SE. ${ }^{*} p<0.01$, significantly different from the indicated group.

\section{Discussion}

The present report provides a systematic analysis of the translation of surface coarseness into neuronal discharges during passive and active whisker movements. We found that the whiskers do more than merely transmit the distance profiles of the various surfaces through whisker vibrations and TG neuronal discharges; rather, their biomechanical properties influence the representation of surface coarseness, highlighting subtle aspects that may be more perceptually relevant. These effects result in the transmission of high-frequency components and the attenuation of low frequencies in the radial distance profiles of various surfaces by whisker vibrations (Fig. 4). Characterization of these transformations reveals that they are dependent on whisker length (Fig. 8) and surface distance (Fig. 9), and occur during both passive and active whisker movements (Fig. 10). Compatible with these observations, we found that a common feature of whisker motion across sandpaper is a multiplicity of discrete, high-frequency stick-slip events (Fig. 6). These events occur during contact with any surface, its coarseness notwithstanding, and are often fol- 
A

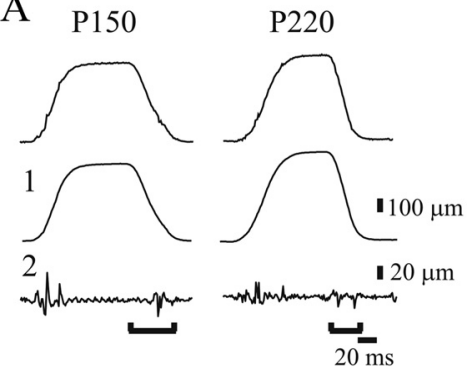

$\mathrm{C}$

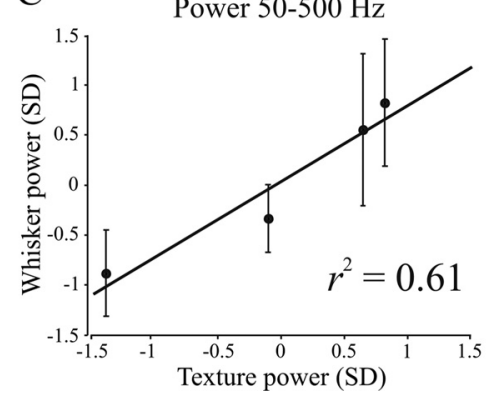

$\mathrm{B}$

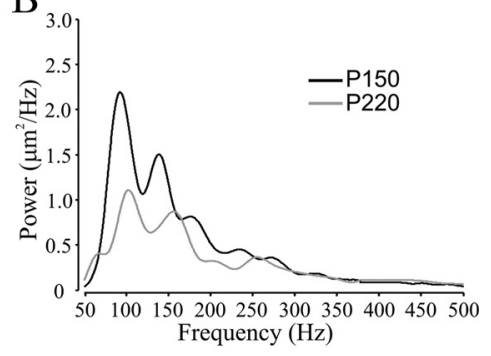

$\mathrm{D}$

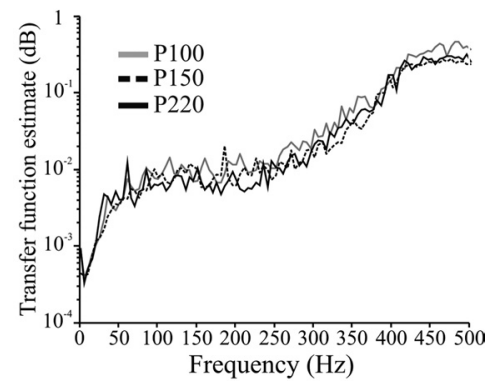

Figure 10. Transmission of surface distance profiles into whisker vibrations during active whisking. $A$, Sample whisker trajectories associated with two surfaces (top). The traces in the middle were filtered (see Materials and Methods) to yield the whisk signal (1) and texture-related whisker trajectories (2). $\boldsymbol{B}$, Average power spectra of B2 whisker movements across the two textures. C, Correlation between power $50-500 \mathrm{~Hz}$ in the distance profiles of different surfaces and whisker vibration signal. D, Transfer function of the two signals across several surfaces. Error bars in the figure indicate the SE. ${ }^{*} p<0.01$ Significantly different from the indicated group.

lowed by high-frequency transient ringing at each whisker's resonance frequency (Fig. 6). These events have also been observed during artificial whisking in anesthetized rats (Arabzadeh et al., 2005, 2006; Lottem and Azouz, 2008) as well as whisking across surfaces in awake behaving conditions (Ritt et al., 2008; Wolfe et al., 2008; Jadhav et al., 2009), and are therefore likely to constitute basic common elements of the whisker sensory input.

We propose, based on our results and others, that the interactions of whiskers with textured surfaces and the resultant TG neuronal discharge are stochastic in nature. Surface coarseness is expressed by the probability of stick-slip events during whisker movement across textures. This probability is determined by the height of surface microfeatures and the biomechanical characteristics of each whisker that influence the bandpass properties of these transformations. This simplifies the problem of texture identification, as it requires the extraction, or decoding, of only a single variable-probability of stick-slip events.

\section{Coding of surface coarseness}

Rats use their whiskers, among other purposes, to discriminate surface coarseness (Guic-Robles et al., 1989; Carvell and Simons, 1990, 1995; Guic-Robles et al., 1992; Ritt et al., 2008). Two major models for coding of surface coarseness in the somatosensory system have been proposed. The resonance model posits that a gradient of biomechanical properties of whiskers leads to a spatial representation of textures. This model makes several predictions that were tested in the current study. First, each whisker displays a fundamental resonance frequency, which varies with whisker length (Hartmann et al., 2003; Neimark et al., 2003). We found that whiskers exhibit high-frequency vibrations during impulse response (Fig. 5), and that the spectral composition of these vibrations varies with whisker length. The frequencies we found were consistent with those encountered using piezoelectric stimulation to the whisker tip (Neimark et al., 2003; Andermann et al.,
2004), but were higher than those found in awake whisking animals (Wolfe et al., 2008) or in plucked whiskers in air (Hartmann et al., 2003; Neimark et al., 2003) by a factor of $\sim 2$. This may result from our experimental setup, i.e., an object moving at a velocity corresponding to head movements and touching the tips of the whiskers. Second, resonant vibrations also occurred during contact with surfaces, as inferred from the presence of spectral peaks in whisker vibrations at resonant frequencies, with longer whiskers vibrating at lower frequencies and shorter whiskers vibrating at higher frequencies (Fig. 6 ). Third, each whisker is tuned to a specific set of textures, leading to relative resonant vibration amplitude during contact with different textures (see Neimark et al., 2003, their Fig. 8). This conjecture is inconsistent with the literature, showing that the amplitude of whisker-resonant vibrations is independent of surface coarseness (Wolfe et al., 2008) and that all whiskers are able to discriminate between different textures (Lottem and Azouz, 2008). This supposition is also incompatible with our results showing that the amplitude of transient high-frequency ringing at the presumed resonance frequencies are not linearly related to surface coarseness (see supplemental Fig. S2, available at www.jneurosci.org as supplemental material). Furthermore, each whisker was not preferentially excited by a specific set of textures (Fig. 8 E). Rather, shorter, anterior whiskers transmitted higher frequencies compared with longer, posterior whiskers (Fig. 4C). This difference was manifested as a somatotopic map of firing rates across the pad (Fig. $4 D$ ), and may also be manifested as a gradient map in somatosensory cortex (S1). Fourth, resonant whisker vibrations serve to amplify specific features in a set of textures that results in neuronal discharges. Transient highfrequency ringing at the presumed resonance frequencies usually occurred after discrete high-velocity stick-slip events and did not lead to neuronal discharge (Fig. 6). Furthermore, when applying an impulse to each whisker, the resulting decaying resonant oscillation did not lead to neuronal discharges (Fig. 5). Finally, resonant whisker vibrations could facilitate the detection of small-amplitude vibrations at the corresponding NF (Andermann and Moore, 2008). Our results do not rule out conclusively the possibility that resonant behavior may amplify stick-slip events that lead to neuronal discharge. However, stick-slip events occur at lower and higher frequencies, respectively, than the NF (Fig. 7). Together, these findings suggest that surface coarseness is not encoded by relative vibration amplitude across facial whiskers, at least in our experimental setup. Nevertheless, the occurrence of whisker resonance during passive and active whisker movements suggests that it may play a role in amplifying some types of whisker responses (Andermann and Moore, 2008; Shatz and Christensen, 2008).

The alternative theory suggests that surface coarseness is translated into distinct kinetic signature in each whisker (Arabzadeh et al., 2003, 2005; Hipp et al., 2006; Lottem and Azouz, 2008; Wolfe et al., 2008). The current study demonstrates that stick-slip events are fundamental elements of natural whisker-surface in- 
teractions, and surface coarseness information is carried mainly by the rate of discrete high-velocity stick-slip events. These conclusions mesh well with earlier studies in anesthetized rats (Jones et al., 2004; Arabzadeh et al., 2005, 2006; Lottem and Azouz, 2008), in awake behaving rats (von Heimendahl et al., 2007; Jadhav et al., 2009), and in primates (Darian-Smith et al., 1982; Sinclair and Burton, 1991; Tremblay et al., 1996), all showing that surface coarseness is coded by firing rate. We show in Figures $2 E$ and $4 C$ that high-frequency whisker vibrations are correlated with surface coarseness. These variations in high-frequency whisker vibrations (Fig. 5) may be the result of changes in the amplitude and the number of high-velocity vibrations (slips). Although both of these parameters may vary across surfaces, in the current study, we examine which of these parameters are relevant to spike discharge. We find that the velocity and amplitude of slips that resulted in spike discharge did not depend on surface coarseness (supplemental Fig. S1 A, B, available at www. jneurosci.org as supplemental material). Since spikes take place mostly during high-frequency events (slips) and their discharge rates are related to surface coarseness, we claim that the rate of slip events that causes neurons to discharge varies with texture. Thus, differences between texture coarseness are not encoded by relative stick-slip event amplitude but rather by their rate.

\section{Active whisking}

Rats discriminate surface coarseness using their whiskers. They do so by moving their whiskers in a rhythmical motion against objects (Welker, 1964; Carvell and Simons, 1990; Berg and Kleinfeld, 2003; Wolfe et al., 2008) and by head motions, too (Carvell and Simons, 1990; Towal and Hartmann, 2006; Mitchinson et al., 2007). Recent studies and the current study show that stick-slip events are a prominent component of whisker motions against surfaces, and that the rate and magnitude of these events correlate well with surface coarseness (Lottem and Azouz, 2008; Wolfe et al., 2008). Our data suggest that the translations of highfrequency components in the radial distance profiles of the various surfaces into whisker vibrations are similar during both active and passive whisker motions (Fig. 10).

The vibrations induced in a whisker as it sweeps over a surface are likely to depend, in addition to the structure of the textured surface, on both intrinsic and extrinsic variables such as features of active whisking (whisk-to-whisk variability, whisking frequency, sweep amplitude, whisker mechanical properties, whisker exploration strategy, etc.), or the distance between whisker shaft and the surface (see a discussion of these issues in Lottem and Azouz, 2008). We suggest, based on our results and the literature (Lottem and Azouz, 2008; Ritt et al., 2008; Wolfe et al., 2008), that because of large variability in the translation of surface coarseness into vibration signals, and the influence of numerous variables on this translation, several strategies in texture coding may be ruled out. First, variation in surface position and whisker velocity (Carvell and Simons, 1990) will alter the NF of each whisker and the "tuning" of the whiskers to the various textures, making it difficult to construct labeled frequency lines in the cortex. Second, whisk-to-whisk variability in the interaction between whiskers and surfaces makes a coding strategy in which the temporal pattern of high-velocity micromotions is preserved in the temporal pattern of spikes (Arabzadeh et al., 2005, 2006) immutable. Thus, the occurrence of stick-slips events is likely to be encoded by discharge rates in $\mathrm{S} 1$ ensembles.

\section{References}

Andermann ML, Moore CI (2008) Mechanical resonance enhances the sensitivity of the vibrissa sensory system to near-threshold stimuli. Brain Res 1235:74-81.

Andermann ML, Ritt J, Neimark MA, Moore CI (2004) Neural correlates of vibrissa resonance; band-pass and somatotopic representation of highfrequency stimuli. Neuron 42:451-463.

Arabzadeh E, Petersen RS, Diamond ME (2003) Encoding of whisker vibration by rat barrel cortex neurons: implications for texture discrimination. J Neurosci 23:9146-9154.

Arabzadeh E, Panzeri S, Diamond ME (2004) Whisker vibration information carried by rat barrel cortex neurons. J Neurosci 24:6011-6020.

Arabzadeh E, Zorzin E, Diamond ME (2005) Neuronal encoding of texture in the whisker sensory pathway. PLoS Biol 3:155-165.

Arabzadeh E, Panzeri S, Diamond ME (2006) Deciphering the spike train of a sensory neuron: counts and temporal patterns in the rat whisker pathway. J Neurosci 26:9216-9226.

Berg RW, Kleinfeld D (2003) Rhythmic whisking by rat: Retraction as well as protraction of the vibrissae is under active muscular control. J Neurophysiol 89:104-117.

Bermejo R, Vyas A, Zeigler HP (2002) Topography of rodent whisking-I. Two-dimensional monitoring of whisker movements. Somatosens Mot Res 19:341-346.

Brecht M, Preilowski B, Merzenich MM (1997) Functional architecture of the mystacial vibrissae. Behav Brain Res 84:81-97.

Brown AW, Waite PM (1974) Responses in the rat thalamus to whisker movements produced by motor nerve stimulation. J Physiol 238:387-401.

Carvell GE, Simons DJ (1990) Biometric analyses of vibrissal tactile discrimination in the rat. J Neurosci 10:2638-2648.

Carvell GE, Simons DJ (1995) Task- and subject-related differences in sensorimotor behavior during active touch. Somatosens Mot Res 12:1-9.

Darian-Smith I, Sugitani M, Heywood J, Karita K, Goodwin A (1982) Touching textured surfaces: cells in somatosensory cortex respond both to finger movement and to surface features. Science 218:906-909.

Fend M, Bovet S, Yokoi H, Pfeifer R (2003) An active artificial whisker array for texture discrimination. Proceedings of the IEEE/RSJ International Conference on Intelligent Robots and Systems (IROS) 2:1044-1049.

Guic-Robles E, Valdivieso C, Guajardo G (1989) Rats can learn a roughness discrimination using only their vibrissal system. Behav Brain Res 31:285-289.

Guic-Robles E, Jenkins WM, Bravo H (1992) Vibrissal roughness discrimination is barrel cortex-dependent. Behav Brain Res 48:145-152.

Hartmann MJ, Johnson NJ, Towal RB, Assad C (2003) Mechanical characteristics of rat vibrissae: resonant frequencies and damping in isolated whiskers and in the awake behaving animal. J Neurosci 23:6510-6519.

Hipp J, Arabzadeh E, Zorzin E, Conradt J, Kayser C, Diamond ME, König P (2006) Texture signals in whisker vibrations. J Neurophysiol 95:1792-1799.

Jadhav SP, Wolfe J, Feldman DE (2009) Sparse temporal coding of elementary tactile features during active whisker sensation. Nat Neurosci. Advance online publication. Retrieved May 31, 2009. doi:10.1038/nn.2328.

Jones LM, Depireux DA, Simons DJ, Keller A (2004) Robust temporal coding in the trigeminal system. Science 304:1986-1989.

Knutsen PM, Derdikman D, Ahissar E (2005) Tracking whisker and head movements in unrestrained behaving rodents. J Neurophysiol 93:2294-2301.

Krupa DJ, Matell MS, Brisben AJ, Oliveira LM, Nicolelis MA (2001) Behavioral properties of the trigeminal somatosensory system in rats performing whisker-dependent tactile discriminations, J Neurosci 21:5752-5763.

Leiser SC, Moxon KA (2006) Relationship between physiological response type (RA and SA) and vibrissal receptive field of neurons within the rat trigeminal ganglion. J Neurophysiol 95:3129-3145.

Lottem E, Azouz R (2008) Dynamic translation of surface coarseness into whisker vibrations. J Neurophysiol 100:2852-2865.

Mehta SB, Kleinfeld D (2004) Frisking the whiskers: patterned sensory input in the rat vibrissa system. Neuron 41:181-184.

Milani H, Steiner H, Huston JP (1989) Analysis of recovery from behavioral asymmetries induced by unilateral removal of vibrissae in the rat, Behav Neurosci 103:1067-1074 
Mitchinson B, Martin CJ, Grant RA, Prescott TJ (2007) Feedback control in active sensing: rat exploratory whisking is modulated by environmental contact. Proc Biol Sci 274:1035-1041.

Moore CI (2004) Frequency-dependent processing in the vibrissa sensory system J Neurophysiol 91:2390-2399.

Moore CI, Andermann ML (2005) The vibrissa resonance hypothesis, in Neural plasticity in adult somatic sensory-motor systems (Ebner FF, ed), pp 21-60, Boca Raton, FL: CRC.

Neimark MA, Andermann ML, Hopfield JJ, Moore CI (2003) Vibrissa resonance as a transduction mechanism for tactile encoding. J Neurosci 23:6499-6509.

Oppenheim AV, Schafer RW (1999) Discrete-time signal processing. Upper Saddle River, NJ: Prentice-Hall.

Ritt JT, Andermann ML, Moore CI (2008) Embodied information processing: vibrissa mechanics and texture features shape micromotions in actively sensing rats. Neuron 57:599-613.

Sachdev RN, Sellien H, Ebner F (2001) Temporal organization of multiwhisker contact in rats. Somatosens Mot Res 18:91-100.

Semba K, Egger MD (1986) The facial "motor" nerve of the rat: control of vibrissal movement and examination of motor and sensory components. J Comp Neurol 247:144-158.

Shatz LF, Christensen CW (2008) The frequency response of rat vibrissae to sound. J Acoust Soc Am 123:2918-2927.

Shoykhet M, Doherty D, Simons DJ (2000) Coding of deflection velocity and amplitude by whisker primary afferent neurons: implications for higher level processing. Somatosens Mot Res 17:171-180.

Sinclair RJ, Burton H (1991) Neuronal activity in the primary somatosensory cortex in monkeys (Macaca mulatta) during active touch of textured surface gratings: responses to groove width, applied force, and velocity of motion. J Neurophysiol 66:153-169.

Stüttgen MC, Rüter J, Schwarz C (2006) Two psychophysical channels of whisker deflection in rats align with two neuronal classes of primary afferents. J Neurosci 26:7933-7941.

Szwed M, Bagdasarian K, Ahissar E (2003) （2003） Encoding of vibrissal active touch. Neuron 40:621-630.

Towal RB, Hartmann MJ (2006) Right-left asymmetries in the whisking behavior of rats anticipate head movements, J Neurosci 26:8838-8846.

Tremblay F, Ageranioti-Bélanger SA, Chapman CE (1996) Cortical mechanisms underlying tactile discrimination in the monkey. I. Role of primary somatosensory cortex in passive texture discrimination. J Neurophysiol 76:3382-3403.

von Heimendahl M, Itskov PM, Arabzadeh E, Diamond ME (2007) Neuronal activity in rat barrel cortex underlying texture discrimination. PLoS Biol 5:2696-2708.

Welker WI (1964) Analysis of sniffing of the albino rat. Behaviour 12:223244.

Wolfe J, Hill DN, Pahlavan S, Drew PJ, Kleinfeld D, Feldman DE (2008) Texture coding in the rat whisker system: slip-stick versus differential resonance. PLoS Biol 6:1661-1677. 